\title{
TEORÍAS DE LA COMPLEJIDAD Y CIENCIAS SOCIALES Nuevas Estrategias Epistemológicas y Metodológicas
}

\author{
Leonardo G. Rodríguez Zoya ${ }^{1}$ \\ Universidad de Buenos Aires, CONICET \\ Julio Leónidas Aguirre ${ }^{2}$ \\ Universidad Nacional de San Martín, Argentina
}

Resumen.- El estudio de la complejidad y de los sistemas complejos ha devenido, desde mediados del siglo XX, en un objeto de estudio central para la ciencia contemporánea pero también para la reflexión filosófica, ética y política. El objetivo de este trabajo es realizar una articulación crítica entre dos modos distintos y antagónicos, quizás, de entender y estudiar la complejidad: el pensamiento complejo de Edgar Morin y las así llamadas ciencias de la complejidad. El artículo explora las implicancias y desafíos epistemológicos y metodológicos que las teorías de la complejidad plantean para la investigación en ciencias sociales.

Palabras clave.- pensamiento complejo, ciencias de la complejidad, epistemología, metodología, modelos basados en agentes

Abstract.- Complexity and complex systems have emerged, around the middle of twentieth century, as a central research object for contemporary science, but also for philosophical, ethical and political reflection. The aim of this work is to make a critical link between two alternative ways, which also can be conceived as antagonistic, of understanding and studying complexity: complex thought proposed by Edgar Morin and the so-called complexity science. The article explores the epistemological and methodological implications and challenges of complexity theories for social sciences research.

Keywords.- complex thought, complexity science, epistemology, methodology, agent-based modeling

\footnotetext{
${ }^{1}$ Licenciado en Ciencia Política por la Universidad de Buenos Aires. Diploma de Honor. Doctorando en Ciencias Sociales por la Universidad de Buenos Aires (Argentina) y Doctorando en Sociología por la Universidad de Toulouse-1 (Francia). Becario de Investigación Doctoral del Consejo Nacional de Investigaciones Científicas y Técnicas de Argentina (CONICET). Cofundador y Coordinador de la Comunidad de Pensamiento Complejo (CPC) (www.pensamientocomplejo.com.ar). Coordinador del Grupo de Estudios Interdisciplinarios sobre Complejidad y Ciencias Sociales (GEICCS). Profesor de metodología de la investigación en ciencias sociales, Facultad de Ciencias Sociales, Universidad de Buenos Aires. Líneas de investigación: pensamiento complejo, teoría de los sistemas complejos, ciencias de la complejidad, epistemología genética, epistemología política, epistemología de las ciencias sociales, metodología de las ciencias sociales. Datos de contacto del autor: mail. leonardo.rzoya@gmail.com | web. www.pensamientocomplejo.com.ar | Tel. (054)-(011)-46245414 | Movil. (54)-(911)-5001-8099

${ }^{2}$ Licenciado en Ciencia Política y Licenciado en Administración Pública por la Universidad Nacional de Cuyo (2009). Mención de Honor Universidad Nacional de Cuyo. Doctorando en Ciencia Política por la Universidad Nacional de San Martín (Argentina). Presidente del Centro de Estudios para la Gestión de Sistemas Complejos (CEGeSCo) (www.cegesco.com.ar). Miembro de la Comunidad de Pensamiento Complejo. Investigador del Centro Interdisciplinario para el Estudio de Políticas Públicas (CIEPP) (www.ciepp.org.ar).
} 


\section{Introducción}

El objetivo de este trabajo consiste en reflexionar sobre el concepto de complejidad organizada en un nivel epistemológico y metodológico, a la luz de los aportes de las teorías contemporáneas de la complejidad. El estudio de los problemas de complejidad organizada ha sido una de las preocupaciones centrales en diversas teorías de complejidad formuladas en el marco de diversas ciencias y disciplinas, como la sistémica, la cibernética, la biología, la termodinámica, la epistemología, la antropología y la investigación social de segundo orden, entre otras.

En el plano epistemológico, el propósito del trabajo consiste en buscar un punto de articulación y complementariedad entre dos modos de abordaje a la complejidad organizada: el pensamiento complejo y las ciencias de la complejidad. En el plano metodológico, se aborda una técnica específica de las ciencias de la complejidad: la simulación basada en agentes. En este marco, se presenta la plataforma informática SocLab, una implementación de software desarrollada sobre la base de la formalización de la sociología de la acción organizada que tiene como finalidad, la modelización y simulación de organizaciones sociales. Se examinan críticamente los resultados de una investigación empírica desarrollada en Francia, la cual empleó la metodología SocLab en el campo de la gobernanza medioambiental del agua.

\section{Un lugar para la complejidad en el pensamiento científico contemporáneo}

La 'complejidad' constituye una perspectiva novedosa y marginal en la ciencia contemporánea; su carácter de novedad radica en que el estudio de la complejidad implica, en buena medida, un quiebre o discontinuidad en la historia de la ciencia o, más precisamente dicho, en la racionalidad científica occidental. La complejidad introduce, en el terreno de las ciencias, una racionalidad post-clásica que habilita e incorpora problemas ignorados o vedados por el pensamiento científico moderno. Estos problemas involucran, en un sentido no exhaustivo, cuestiones relativas al desorden, el caos, la no-linealidad, el no-equilibro, la indecibilidad, la incertidumbre, la contradicción, el azar, la temporalidad, la emergencia, la auto-organización. La complejidad puede entenderse, por lo tanto, como un paradigma científico emergente que involucra un nuevo modo de hacer y entender la ciencia, extendiendo los límites y criterios de cientificidad, más allá de las fronteras de la ciencia moderna, ancladas sobre los principios rectores del mecanicismo, el reduccionismo y el determinismo (Delgado Díaz 2004; Morin 2004b; Sotolongo y Delgado Díaz 2006; Vilar 1997).

Por otro lado, la complejidad se ubica en una zona marginal del saber científico contemporáneo, aunque sin duda sus grados de penetración, y por consiguiente de marginalidad y desconocimiento, varían de una ciencia y/o disciplina a otra. Para decirlo lisa y llanamente, las teorías y métodos asociados a 'la complejidad' no constituyen el mainstream en los campos científicos o disciplinares en los que se desarrollan. Esta situación de marginalidad es menor en el campo de las ciencias de la materia y de las ciencias de vida, campos en los cuales es posible rastrear la prehistoria de las ideas científicas de lo que hoy se denomina 'teoría/s' y/o 'ciencias' de 
la complejidad ${ }^{3}$. El hecho relativo a que estas ciencias hayan sido más receptivas del estudio científico de la complejidad obedece también a otro hecho no menos significativo, el cual refiere al lenguaje propio en el que se expresa el pensamiento de las ciencias físico-naturales, más proclive o sensible al empleo del lenguaje formal y matemático. Así, el estudio y dominio de la complejidad en el terreno científico (por distinción -mas no oposición- respecto del pensamiento filosófico y del lenguaje natural más comúnmente empleado en las disciplinas humanísticas y sociales) ha estado estrechamente ligado a la invención y el desarrollo de la computación moderna ${ }^{4}$. De este modo, la teoría matemática de la complejidad y las ciencias de la computación ${ }^{5}$ constituyen el andamiaje necesario, pero no exclusivo ni exhaustivo, para el abordaje de una mirada científica de la complejidad en el campo de las ciencias de la vida y de la materia en sentido amplio (física, química, biología, termodinámica, etc.).

Lo que hoy suele llamarse 'teoría de la complejidad' -en singular-, o en su denominación más pluralista, 'teorías de la complejidad' -en plural-, es en realidad el nombre de un campo con límites borrosos que abarca, en su formulación científica, a las teorías de los sistemas complejos en sentido amplio (sistemas dinámicos, sistemas no lineales, sistemas adaptativos), la teoría del caos y los fractales ${ }^{6}$ (Morin 2004a; Reynoso 2009). Lo cierto es que no existe en la actualidad, una teoría unificada de la complejidad, que sintetice y sistematice de modo explícito los aspectos fundamentales de las distintas y variadas teorías, métodos y algoritmos de complejidad elaborados en el marco de ciencias y disciplinas disímiles ${ }^{7}$.

\footnotetext{
${ }^{3}$ Esta cartografía de los antecedentes científicos de la complejidad está aún por escribirse, pero a título indicativo y no exhaustivo, pueden enumerarse algunos hitos. La teoría de la evolución de Charles Darwin, y la termodinámica clásica -ciencia del calor- introduce el problema de la temporalidad, ausente por completo en el marco de la física newtoniana, de carácter universal y, por lo tanto, atemporal y ahistórica. Sendas teorías, introducen el problema del tiempo en el pensamiento científico en dos sentidos opuestos. Una, la teoría de la evolución, hace referencia a la emergencia y creación de nuevas estructuras más complejas a lo largo de un proceso diacrónico; la otra, la termodinámica, introduce una flecha del tiempo contraria, el paso del tiempo se asocia a la destrucción de las estructuras pre-existentes (entropía). A estas referencias cabría adicionar: (a) la revolución cuántico-relativista en la física de finales del siglo XIX y las tres primeras décadas del siglo $\mathrm{XX}$, incluyendo aquí el principio de complementariedad onda-corpúsculo de Bohr y el principio de incertidumbre de Heisenberg; (b) la formulación de las lógicas no-clásicas que intentan superar los principios de identidad, no contradicción y tercio excluso de la lógica clásica (Gödel, Tarski, Da Costa, Zaeh, entre otros).

${ }^{4}$ Los antecedentes de la ciencia de la computación, se retrotraen a las contribuciones pioneras del británico Alan Turing, quien desarrolló uno de los primeros modelos computacionales, que se conoce como máquina de Turing. También, contribuyó a la codificación del "inviolable" código alemán Enigma, durante la Segunda Guerra Mundial. Puede considerarse como uno de los pioneros en el estudio de la emergencia por sus contribuciones en el campo de la biología matemática y la morfogénesis. En 1952 publicó un trabajo titulado Fundamentos Químicos de la Morfogénesis en donde indaga en la construcción de órdenes o patrones complejos a partir de reglas y patrones sencillos (Johnson 2001:39-40). Fue víctima de las leyes homofóbicas británicas, en 1952 fue procesado por homosexualidad y condenado a elegir entre la esterilización química o la cárcel. Se suicidó en 1954, comiendo una manzana a la cual le había inyectado cianuro. Décadas más tardes, la compañía Apple Macintosh emplearía como logo una manzana mordida, en honor a Alan Turing.

${ }^{5}$ Sobre las matemáticas de la complejidad y su relación con las ciencias de la complejidad y las ciencias sociales, véase (Maldonado 2008, 2009).

${ }^{6}$ No hay consenso acerca de si la geometría fractal, formulada por Benoît Mandelbrot (1987), forma o no parte de este campo. Para una discusión sobre este punto véase, Reynoso (2006).

${ }^{7}$ Este hecho es reconocido por varios autores del campo. Carlos Reynoso se pronuncia del siguiente modo: "no existe nada que se asemeje a una teoría unificada o a un conjunto de acuerdos sustanciales, y al lado de hallazgos espectaculares subsisten fuertes dudas sobre la practicabilidad
} 
La complejidad como objeto de reflexión ha estado ausente de los grandes debates de la filosofía de la ciencia del siglo XX; de Viena a Popper, y de éstos a la filosofía post-empirista (post-kuhniana y post-popperiana), de hecho la complejidad no ha sido una cuestión pensada y debatida por los grandes referentes de la filosofía de la ciencia, como si lo ha sido, la verdad, la inducción, la racionalidad, los valores (Morin 1990). La complejidad, en tanto problema, conjunto de teorías y métodos y, en un sentido más general, como campo de estudios de la ciencia contemporánea, o mejor aún, como un paradigma científico emergente, es incluso más marginal en las ciencias sociales y humanísticas. La epistemología y metodología de las ciencias sociales, de un lado; y las teorías contemporáneas de la complejidad, del otro, son dos mundos con escasos puntos de conexión y con pocos puentes articuladores ${ }^{8}$.

\section{Los problemas de complejidad organizada}

El término complejidad organizada fue acuñado por Warren Weaver (1948) en un artículo publicado bajo el emblemático título "Sience and Complexity" , y que puede considerarse con justicia, como una de las contribuciones fundacionales del campo, en la que se emplea por primera vez el término complejidad de modo deliberado y explícito. No es exagerado decir que con el texto de Weaver, el 'significante' complejidad irrumpe en el vocabulario científico, lo que permitirá, décadas más tardes, dotar de identidad, a un conjunto de teorías formuladas en distintas disciplinas y ciencias.

En dicho artículo Weaver bosqueja una tipología de problemas científicos a partir de la historia de las ciencias en los últimos tres siglos. Distingue así entre los problemas de simplicidad, los problemas de complejidad desorganizada y, finalmente, los

de buena parte del proyecto" (Reynoso 2006:12). No obstante estos pronósticos, y reconociendo de hecho la heterogeneidad de formulaciones teóricas y técnicas sumamente especializadas en el estudio de fenómenos complejos, es digno de destacar el trabajo de Carlos Eduardo Maldonado, quien desde una óptica de filosofía de la ciencia, se ha atrevido a plantear el problema de una teoría general de la complejidad (Maldonado 2007:101-132).

${ }^{8}$ No obstante, y para ser justos con la historia, es preciso introducir una afirmación hipotética que matice la afirmación realizada. Si bien la complejidad constituye una novedad en la historia de la ciencia contemporánea, e incluso puede afirmarse que constituye 'un nuevo tipo de ciencia'; los problemas a los que ella refiere, -y en un sentido más general, las propiedades con las que suelen caracterizarse a los sistemas complejos (evolución no lineal, auto-organización, emergencia, transiciones orden/caos, continuidad y el cambio de estructuras, entre otros)-, han sido cuestiones tematizadas bajo otras denominaciones por distintas tradiciones de las ciencias sociales (Hegel y Marx por ejemplo), y han estado presentes por centurias en la historia del pensamiento filosófico (a partir de Heráclito y Lao Tse). Incluso, algunos planteos de las ciencias de la complejidad pueden asimilarse al pensamiento de las civilizaciones pre-hispánicas de América Latina o a la filosofía oriental. En relación a este último paralelismo, cabe mencionar la obra del físico Fritjof Capra (1984) que lleva el sugerente título El Tao de la física, y que no ha estado exento de críticas. Sería erróneo, sin embargo, tratar como equivalentes a las teorías científicas de la complejidad y las líneas de pensamiento referidas. Sería también ilegítimo, buscar analogías directas y lineales entre los conceptos y términos de una y otra perspectiva. En todo caso, este señalamiento permite precisar una línea de investigación poco explorada aún, la realización de una genealogía de la idea de complejidad en la historia de la filosofía y de la ciencia. El desarrollo de esta indagación permitiría una comparación explícita con el desarrollo de las teorías contemporáneas de la complejidad.

${ }^{9}$ El artículo fue publicado en la revista American Scientist $n^{\circ} 36$ en 1948. Constituye, en realidad, un extracto del capítulo1 del libro "The Scientists Speak", publicado un año antes por Boni \& Gaer Inc. Dicho libro sintetiza los resultados de una investigación llevada adelante por Weaver y financiada por la Fundación Rockefeller. 
problemas de complejidad organizada. En primer lugar, los problemas de simplicidad corresponden a situaciones que pueden ser descriptas con pocas variables. Su formulación concierne al desarrollo de la ciencia física entre los siglos XVII y XIX, y se vincula directamente con el desarrollo de modelos mecánicos característicos de la física newtoniana. En segundo lugar, Weaver señala que hacia fines del siglo XIX y comienzos del siglo XX cobraron relevancia la teoría de la probabilidad y el desarrollo de la mecánica estadística, las cuales permitieron abordar un nuevo tipo de situaciones caracterizadas por la presencia de un alto número de variables. Si bien el valor de cada elemento / variable individual resulta desconocido o incalculable, el sistema como todo posee cierto orden y resulta analizable en términos de distribuciones promedio. Este segundo tipo de situaciones recibe el nombre de complejidad desorganizada, y resultan abordables por medio de técnicas estadísticas. Finalmente, Weaver llama la atención sobre la existencia de una 'región media', poco explorada por la ciencia, cuya característica esencial no tiene que ver tanto con el número de variables involucradas, sino por el modo en que éstas están relacionadas. La característica fundamental de los fenómenos de esta región media es la organización. Weaver acuña el término de complejidad organizada para referirse a aquellos fenómenos o problemas en donde intervienen un número amplio de factores o variables interrelacionados que conforman un todo orgánico. En otros términos, abordar el problema de la organización implica dar cuenta de la génesis y emergencia de totalidades complejas, que no resultan comprensibles por medio de enfoques reduccionistas-analíticos. Mientras que los problemas de simplicidad pueden ser abordados por modelos mecánicos, y los problemas de complejidad desorganizada pueden ser estudiados por medio de modelos estadísticos; los problemas de complejidad organizada son abordables por modelos sistémicos (Reynoso 2006).

\section{Complejidad organizada y sistemas complejos}

El estudio de los problemas de complejidad organizada ha sido abordado en el marco de campos disciplinares muy distintos, dando lugar a un conjunto de teorías, entre las que cabe destacar a: la cibernética (Wiener 1985), la cibernética de segundo orden (Foerster 1996), la epistemología genética (Piaget 1978), la teoría de la auto-organización (Ashby 1962), la teoría general de los sistemas (Bertalanffy 1968), la geometría fractal (Mandelbrot 1987), la teoría de los autómatas celulares (Neumann 1966, 1968), la termodinámica de los procesos irreversibles (Prigogine y Nicolis 1987), la teoría de la autopoiesis (Maturana y Varela 1972), la teoría de las catástrofes (Thom 1976), entre otras.

La noción de complejidad organizada como totalidad compuesta por elementos heterogéneos articulados entre sí de manera orgánica, remite a la noción de sistema. Así, el modo de abordaje sistémico que reclaman los problemas de complejidad organizada plantea la necesidad de articulación entre tres conceptos fundamentales: complejidad, organización y sistema.

(1) Complejidad y organización: La existencia de fenómenos organizados permite suponer la existencia de un principio opuesto a la dispersión. Es decir, hay organización cuando hay algo que resiste a la dispersión, a la disgregación, a la disolución. Por consiguiente, pensar en el surgimiento y 
mantenimiento de fenómenos organizados, implica pensar en un principio organizador que permita unir y mantener las partes o elementos de una totalidad organizada. La idea de organización remite a la vez a lo organizado (el resultado) y lo organizante (la actividad organizadora) (Morin 1977).

La relación entre complejidad y organización puede plantearse desde dos perspectivas distintas. Desde la perspectiva del pensamiento complejo, Edgar Morin propone comprender la complejidad en términos organizaciones. Se trata de pasar de una noción de objeto esencial/sustancial a una noción de objeto relacional, es decir, de totalidades organizadas compuestas por elementos heterogéneos en interacción. La idea de organización remite así la idea de una totalidad relativa, no cerrada, sino abierta, histórica y contextualizada. Morin destaca que la organización es algo común al mundo físico, biológico y antroposocial y propone pensar la idea de organización a partir de un marcoconcepto que denomina bucle tetralógico, con el cual busca dar cuenta de la relación complementaria-concurrente y antagonista entre los conceptos de orden-desorden-interacciones (encuentros)-organización: "para que haya organización es preciso que haya interacciones: para que haya interacciones es preciso que haya encuentros, para que hay encuentros, es preciso que haya desorden (agitación, turbulencia) (Morin 1977:69).

Desde la perspectiva de las ciencias de la complejidad, se plantea el problema y la necesidad de determinar el nivel de complejidad de un sistema o totalidad organizada. En otros términos, conceptualizar nuestros objetos de estudio como problemas de complejidad organizada, conlleva como corolario, la necesidad de distinguir distintos grados de complejidad de las organizaciones. ¿Qué es lo que hace a una organización más compleja qué otra? Se plantea, por lo tanto, una implicancia metodológica: la medición de la complejidad (Maldonado 2007)

(2) Organización y sistema: La distinción y vinculación entre los conceptos de sistema y organización se encuentra presente en la Teoría General de los Sistemas formulada por Bertalanffy (1968). La noción de sistema remite a la idea de totalidad, de unidad, de conjunto; la cual agrupa, reúne, contiene, a distintos elementos interrelacionados. Morin sugiere vincular la idea de totalidad e interrelación contenidas en la noción de sistema, con la de organización. Así, el sistema puede ser definido como "unidad global organizada de interrelaciones entre elementos, acciones o individuos"; y la organización, como "la disposición de relaciones entre componentes o individuos que produce una unidad compleja o sistema" (Morin 1977:124, 126). Los términos sistema y organización se implican mutuamente, y es imposible concebir uno sin el otro. Si estos así, entonces, tenemos que pensar a los sistemas en términos organizacionales $\mathrm{y}$, al mismo tiempo, pensar las organizaciones en términos sistémicos.

(3) Complejidad y sistemas: La articulación entre estos dos términos es quizás la más difícil de precisar, puesto que constituye el tema con menor tratamiento en la bibliografía especializada. Los autores que se enmarcan en el pensamiento / paradigma sistémico sólo tangencialmente hacen 
referencia a la complejidad. Por otro lado, los autores que trabajan en complejidad, ya sea desde la perspectiva del pensamiento complejo o las ciencias de la complejidad, si bien hacen referencia explícita a los 'sistemas', o más precisamente a los así llamados 'sistemas complejos', rara vez explicitan las diferencias con el pensamiento / paradigma sistémico. En este sentido cabe formular algunas preguntas de difícil respuesta ¿Cuáles son las similitudes y diferencias entre el paradigma sistémico y el paradigma de la complejidad; entre el pensamiento sistémico y el pensamiento complejo, en definitiva, entre la complejidad y los sistemas?

La respuesta a estos interrogantes requiere de una labor filosófica y epistemológica que excede los límites de este trabajo y que, por lo tanto, no nos podremos ocupar aquí. No obstante, el campo de sentido generado por estos interrogantes, permiten introducir unos más concretos y plausibles, a saber: ¿puede haber sistemas no complejos?, y si tal respuesta es afirmativa, ¿cuál es la diferencia entre un sistema complejo y uno no complejo? ¿Qué es lo que hace que un sistema sea más complejo que otro? ¿En qué radica la complejidad de un sistema?

La definición de sistema como totalidad organizada de elementos heterogéneos interrelacionados, no permite distinguir un sistema complejo de un sistema no complejo, lo que podríamos llamar un sistema simple. El interrogante a despejar consiste en saber, cómo es posible distinguir lo que en términos discretos podemos llamar la complejidad y la no complejidad; o lo que en términos de un continuo podemos denominar grados de complejidad de un sistema. Una respuesta posible, consiste en introducir una dimensión cuantitativa en la definición de sistema, a partir de la cual resulta factible afirmar que un sistema complejo es aquel compuesto por muchos elementos y muchas relaciones. Esta definición cuantitativa de complejidad resulta insatisfactoria, porque al asociar la complejidad a una magnitud numérica ${ }^{10}$ de elementos/variables/interacciones, se tornaría imposible el abordaje y la comprensión de esa otra complejidad, fundamental y vital, a saber: la complejidad no cuantitativa. La mayoría de los problemas fundamentales de la civilización contemporánea (hambre, pobreza, desigualdad, degradación de la biósfera, etc.) deberían ser reconocidos como problemas complejos, aunque no tratables y reductibles a términos matemáticos/cuantitativos exclusivamente. En esta línea de reflexión, Edgar Morin propone distinguir los términos de complicación y complejidad, mientras que el primero consiste en una noción fundamentalmente cuantitativa (alto número de interacciones y variables) asociada a los problemas de complejidad desorganizada; la complejidad, por su lado, es planteada más como una noción lógica-cualitativa vinculada con la complejidad organizada (Morin 1990, 2004b). Siguiendo esta distinción podemos inferir que sería posible pensar en sistemas complicados y sistemas complejos.

Si la complejidad, como hemos visto, no depende de una magnitud cuantitativa, entonces, la complejidad de un sistema complejo depende más del tipo de relaciones que de su número. Por consiguiente, puede haber sistemas con

\footnotetext{
${ }^{10}$ Si bien me he referido de modo laxo a esta magnitud cuantitativa en términos de "muchos", sería posible pensar magnitudes matemáticas específicas.
} 
comportamientos complejos que se auto-organizan a partir de reglas e interacciones relativamente simples; para ponerlo en otros términos, la complejidad es la resultante de un proceso de baja complejidad.

Una de las conceptualizaciones epistemológicamente más sólidas de sistemas complejos es la elaborada por Rolando García (2006), quien concibe a los sistemas complejos como totalidades organizadas compuestas por elementos "no separables". El requisito de no separabilidad, conlleva una presunción antireduccionista, ya que si los elementos no son separables, entonces, no pueden ser estudiados de manera aislada. Esta distinción entre separabilidad / no separabilidad permite a su vez distinguir entre dos tipos de sistemas; por un lado, los sistemas descomponibles, por el otro los sistemas no descomponibles. En los primeros, las partes del sistema pueden ser aisladas y estudiadas de modo independiente; mientras que en los segundos, los componentes del sistema están determinados mutuamente. Esta conceptualización le permite a García introducir y precisar el término de interdefinibilidad, el cual supera el concepto de interacción o interrelación. La interdefinibilidad exige que los componentes de un sistema sean definidos y estudiados en función del resto y, por lo tanto, no resulta posible el estudio separado de sus partes (García 2000). En suma, los sistemas complejos son sistemas nodescomponibles cuyos elementos están interdefinidos.

\section{La tensión entre pensamiento complejo y ciencias de la complejidad}

El campo de estudios contemporáneos sobre complejidad se ha desarrollado a partir de dos modos de abordaje distintos: la complejidad restringida y la complejidad general (Morin 2004a). Por un lado, la complejidad restringida, conformada por las llamadas ciencias de la complejidad o ciencias de los sistemas complejos, consiste en una perspectiva que se ha desarrollado notablemente en el mundo anglosajón. Este enfoque puede entenderse como un abordaje fundamentalmente metodológico, técnico y procedimental de la complejidad, basado en la utilización de lenguajes formales, modelos matemáticos y la simulación computacional (Axelrod 1997; GellMann 1994; Gilbert y Troitzsch 1999; Holland 1995). Esta perspectiva provee herramientas metodológicas concretas como los autómatas celulares, las redes booleanas, las redes neuronales adaptativas, los algoritmos genéticos y los sistemas multi-agente, entre otros. Estas herramientas permiten abordar el estudio de los más diversos sistemas complejos: ambientales, sociales, organizacionales, económicos (Maldonado 2007; Reynoso 2006).

Por otro lado, la complejidad general, cristalizada en la propuesta del pensamiento complejo (Morin 1990), se ha desarrollado principalmente en el mundo franco-latino. Este enfoque puede ser definido como una epistemología transdisciplinaria (Morin 1977, 1980, 1986), una filosofía ético-política de la complejidad (Morin 2004c) y, finalmente, un marco epistémico y una cosmovisión orientada hacia la constitución de un paradigma de complejidad (Morin 1991) y una civilización planetaria asentada sobre un desarrollo ético del ser humano, la naturaleza y la biósfera terrestre (Morin 2001; Morin y Brigitte Kern 1993).

Anteriormente he dicho que la complejidad constituye una perspectiva marginal en la ciencia contemporánea, pero es necesario subrayar que al interior del campo de 
estudios sobre complejidad, el mainstream lo constituyen las ciencias de la complejidad, siendo el pensamiento complejo la perspectiva minoritaria dentro del campo. Un académico destacado, se pronuncia en estos términos:

"Existen dos grandes comprensiones de complejidad, usualmente indiferentes entre sí, distantes incluso, y quizás radicalmente distintas. De un lado, la complejidad como ciencia, y de la otra, la complejidad como método. Resulta más apropiado referirnos a la primera como las ciencias de la complejidad o también, más prudentemente, como el estudio de los sistemas complejos adaptativos. En cuanto a la segunda concepción, es conocida genéricamente como el pensamiento complejo. Mientras que la primera hace referencia a diversos, incluso numerosos, autores y líneas de trabajo e investigación, en el segundo caso se trata prioritariamente de la obra de un solo autor, aunque sean numerosos sus seguidores $y$ epígonos" (Maldonado 2007:19).

Mi posición es la siguiente, si el mero criterio cuantitativo no se es suficiente para precisar el grado de complejidad de un sistema, menos aún lo es para clarificar las tensiones y los antagonismos entre estos dos modos de abordaje: ciencias vs. pensamiento de la complejidad. Es decir, el hecho que el pensamiento complejo sea el producto de un solo autor, no invalida su propuesta o planteamiento, pero tampoco los exime de la crítica. Más aún, si las ciencias de la complejidad constituyen la visión hegemónica o dominante dentro del campo, la pregunta inteligente y necesaria consiste en saber por qué esto es así. Es decir, qué intereses rigen la producción de conocimiento en ciencias de la complejidad, y a quiénes benefician los saberes por ellas elaborados.

En trabajos anteriores me he pronunciado sobre la necesidad de redefinir la concepción epistemológica dominante que fundamenta un concepto de ciencia que excluye sistemáticamente la dimensión ético-política del conocimiento científico y de las prácticas científicas. He propuesto, también, la elaboración de una epistemología política, la cual podría contribuir a fundamentar una significación de ciencia no hegemónica abierta al problema político la ciencia (Rodríguez Zoya 2010b).

La distinción más fundamental entre pensamiento complejo y ciencias de la complejidad, no es sólo metodológica, sino también y más fundamentalmente epistemológica y política.

En el nivel metodológico, el pensamiento complejo se propone como un método no clásico que partiendo de una crítica a las metodologías tradicionales en tanto guías a priori de la investigación, apuesta a constituirse como la estrategia de conocimiento de un sujeto quien construye y redefine su estrategia de conocimiento conforme se desarrolla el proceso cognoscitivo. La propuesta del pensamiento complejo ha sido duramente criticada en su formulación metodológica puesto que no provee las herramientas necesarias para conducir satisfactoriamente una investigación empírica. Rolando García se pronuncia en estos términos: "Edgar Morin contribuyó a demoler las bases del racionalismo tradicional que había penetrado tan 
profundamente en el sistema educativo francés. [...] Sin embargo, su crítica no ofrece una formulación precisa de los problemas que enuncia [...] como para conducir a una metodología de trabajo aplicable a las situaciones concretas que él considera como 'complejas"' (García 2006:21). No obstante estas agudas críticas, cabe aclarar que el fin de la teorización de Edgar Morin sobre el método, no consiste proveer útiles metodológicos-procedimentales, y mucho menos aún técnicos, que sus críticos le acusan de carecer. Otra de las observaciones agudas que jalonan la crítica a la obra moriniana, consiste en destacar que la elaboración moriniana de la complejidad constituye un paradigma o teoría discursiva que toma a la complejidad como objeto mismo de reflexión, a la cual se llega por la vía del lenguaje natural y metafórico (Reynoso 2006, 2009). Esta crítica es disparada, claro está, desde el terreno propio de las ciencias de la complejidad, las cuales recurren para su instrumentalización técnica al empleo de lenguajes formales, matemáticos y computacionales. Pero incluso, es necesario decirlo, las ciencias que emplean lenguajes formales son también una clase de discurso; o mejor expresado aún, toda teoría científica puede ser concebida como una sub-clase del discurso científico.

Por otro lado, las ciencias de la complejidad se han desarrollado a partir de la formulación de metodologías y técnicas sofisticadas para abordar el estudio de sistemas complejos. Es decir, estas ciencias poseen un poderoso arsenal de tipo técnico instrumental, sustentado en el poder de cálculo de la computación contemporánea, para abordar el estudio formal y aplicado de sistemas complejos.

Por consiguiente, el balance metodológico de la tensión pensamiento complejo ciencias de la complejidad puede plantearse del siguiente modo. El pensamiento complejo intenta vertebrar un método no clásico para el estudio de la complejidad, éste método atribuye de modo ineludible un rol central al sujeto de conocimiento en la elaboración de su estrategia cognitiva. Pero más aún, el sujeto del pensamiento complejo no es meramente un sujeto reducido a su dimensión epistémica-racional; por el contrario, es un sujeto abierto a la complejidad humana. Así entendida, la complejidad generalizada, trasciende los límites del quehacer científico y concierne también "a nuestro conocimiento como ser humano, individuo, persona y ciudadano", dice Morin. La complejidad "es un cierto número de principios que ayudan al espíritu autónomo a conocer" (Morin 2004a:48). La metodología de las ciencias de la complejidad, específicamente la modelización y simulación de sistemas complejos, constituye una tercera vía de hacer ciencia, distinta y complementaria a la deducción y la inducción (Axelrod 2004; Maldonado y Gómez Cruz 2010). No obstante, el concepto de método científico que estas nuevas ciencias defienden, comparte la matriz clásica de método: herramientas y procedimientos neutrales, impersonales, universales y repetibles. La oposición real en el plano metodológico entre el pensamiento complejo y las ciencias de la complejidad, concierne al lugar del sujeto en la metodología científica. El pensamiento complejo reclama la necesidad de incorporar de modo sistemático y explícito la subjetividad reflexiva en la construcción de la ciencia; las ciencias de la complejidad borran al sujeto de su formulación metodológica y epistemológica.

Así, la indagación metodológica nos ha trasladado al nivel del marco epistemológico en el que se sustentan ambas formulaciones. Para expresarlo de modo sintético y sin rodeos; el pensamiento complejo busca elaborar un marco epistemológico ampliado en donde el conocimiento científico pueda ser articulado y puesto en 
relación con otras formas de conocimiento no científico, la filosofía, y los saberes humanísticos: la literatura, el cine, la poesía, el arte. En el terreno científico, la vocación religadora del pensamiento complejo busca establecer los puentes comunicantes entre las ciencias físicas, las ciencias de la vida y las ciencias antropo-sociales; una búsqueda de tipo transdisciplinaria que permitiría conducir a una nueva organización de los saberes por medio de la reelaboración de los principios rectores del pensamiento. Así, el pensamiento complejo "está animado por una tensión permanente entre la aspiración a un saber no parcelado, no dividido, no reduccionista, y el reconocimiento de lo inacabado e incompleto" (Morin 1990:23). La propuesta del pensamiento complejo desarrollada por Morin consiste en un replanteo epistemológico que lleva a una nueva organización del conocimiento, tanto a nivel personal como social e institucional. Se trata de una estrategia meta-cognitiva que tiene por finalidad reformar los principios matriciales del pensamiento simplificador (disyunción y reducción) que llevaron a la instauración de las dicotomías fundantes de la matriz de pensamiento occidental: sujeto / objeto; mente / cuerpo; cultura / naturaleza; filosofía / ciencia; valor / hecho; afectividad / razón. Así, el pensamiento complejo reclama la constitución de un saber pertinente, ecologizado, histórico, contextual. El replanteo epistemológico que formula el pensamiento complejo se extiende, al mismo tiempo, más allá de la dimensión epistémica de la ciencia y desemboca en una propuesta ético-política: el desarrollo de una política planetaria de civilización, la constitución de una ética planetaria y el pleno empleo de las potencialidades humanas para civilizar nuestras ideas. La conjunción de estas tres vías permitiría, según Morin, salir de la edad de hierro planetaria y de la prehistoria del espíritu, para dar a luz a la humanidad de la humanidad, es decir, el surgimiento de una verdadera ciudadanía planetaria, la Tierra-Patria. Podemos ver que la complejidad general, en la propuesta del pensamiento complejo, desemboca en una reforma de la civilización occidental.

Por el contrario, las ciencias de la complejidad, pese a sus avances tecnológicosinstrumentales de punta, están enraizadas en una epistemología pobre, estrecha y limitada, que impide conectar de modo explícito el saber científico, con la filosofía, la política, la ética y la vida. Los supuestos epistemológicos que sustentan a las nuevas ciencias de la complejidad no permiten dar cuenta de la dimensión ético-política constitutiva y condicionante de toda práctica científica. Se convierten así, bajo el halo de una supuesta neutralidad ideológica, en herramientas con profunda significación política, casi siempre funcionales a los intereses del norte.

Para expresarlo de modo claro y directo, pensamiento complejo y ciencias de la complejidad son dos modos de abordaje que se ignoran mutuamente con pocos puntos de contacto sistemáticos y explícitos. Tomados aisladamente cada uno de ellos es en sí mismo insuficiente. Mirados con ojos críticos, el pensamiento complejo presenta una hipertrofia filosófica, es como un cuerpo con una gran cabeza pero con manos pequeñas: puede pensar y decir mucho, pero hacer poco. Las ciencias de la complejidad presentan una hipertrofia práctica, son como un cuerpo con una cabeza diminuta pero con brazos y manos ágiles y fuertes: pueden hacer mucho, pero pensar poco. Hay una complementariedad necesaria entre pensamiento y ciencias de la complejidad. El pensamiento complejo puede brindar el campo reflexivo necesario para desarrollar un marco epistémico inclusivo de valores éticos y políticos conformes a las necesidad y desafíos de las comunidades sociales, locales, nacionales, regionales y planetaria. Las ciencias de la complejidad pueden brindar 
las herramientas metodológicas concretas para el estudio de fenómenos complejos. Considero que el desafío más fundamental es estimular el desarrollo de las ciencias de la complejidad guiadas por un pensamiento complejo.

\section{Modelización y simulación de sistemas complejos}

Una de las principales estratégicas metodológicas de las ciencias de la complejidad consiste en la modelización y simulación computacional de sistemas complejos. Si bien es posible identificar una pluralidad de significados y usos del concepto de modelo en ciencias (Armatte 2006), el término modelado científico se refiere genéricamente al proceso por el cual se crea una abstracción de un sistema u objeto real, con la finalidad de desarrollar procesos de inferencia sobre los mismos. Los resultados del proceso de inferencia, permiten un análisis e interpretación del sistema u objeto de estudio, lo que retroalimenta el proceso por medio del ajuste/rectificación del modelo en base a los resultados esperados y obtenidos. Si bien es posible construir modelos en base al lenguaje natural, la ciencia usualmente ha desarrollado modelos formulados en lenguajes formales, fundamentalmente las matemáticas.

El modelado científico tradicional estaba basado en ecuaciones matemáticas (ecuaciones diferenciales, procesos estocásticos, entre otros) (Arroyo Méndez y Hassan Collado 2007), la imposibilidad de resolución matemática de un modelo introduce pues, una fuerte limitación. El desarrollo de las ciencias de la computación ha permitido desarrollar nuevas herramientas de cálculo e inferencia: los ordenadores. Así, "la simulación computacional como proceso inferencial" permite el abordaje de "modelos formales [...] intratables matemáticamente" (Izquierdo, et al. 2008).

\section{Modelos basados en agente}

Los modelos basados en agentes (MBA) constituyen una de las técnicas para modelizar sistemas complejos. Junto a los MBA es posible reconocer otros algoritmos de la complejidad que constituyen el heterogéneo y diverso mapa técnicometodológico de las ciencias de la complejidad, a saber: autómatas celulares (AC), redes booleanas aleatorias (RBA), redes neuronales, algoritmos genéticos (AG), entre otros (Gilbert y Troitzsch 1999; Reynoso 2006).

Los antecedentes de la modelización basada en agentes se retrotrae a la década del '40, período en el cual Von Neumann formula su teoría sobre los autómatas celulares (Neumann 1966, 1968). La estructura básica de un autómata celular consiste en una matriz cuadriculada, denominado "espacio celular", en la cual cada celda recibe el nombre de "célula". Al mismo tiempo, cada célula es susceptible de asumir distintos "estados". Por otro lado, cada célula mantiene relaciones de "vecindad" con sus aledañas. El punto interesante a destacar es que el estado que adopta una célula depende del tipo de relación que establezca con sus células vecinas, es decir, el estado de una célula determinada, dependerá de los estados de su vecindad. A este proceso se lo conoce con el nombre de "regla" o "función de transición”. (Arroyo Méndez y Hassan Collado 2007; Reynoso 2006). 
Los modelos basados en agentes permiten dar cuenta de la emergencia y autoorganización de sistemas complejos. Se reconoce que la organización como totalidad (abierta, relativa, cambiante e histórica) es el producto de comportamientos individuales y sus interacciones, es decir, la totalidad organizada (el sistema complejo) es un resultado emergente de patrones de interacción locales. Por consiguiente, los modelos basados en agentes parten de reglas sencillas que especifican el comportamiento individual y la interacción local entre los agentes, con la finalidad de inferir las propiedades globales de todo el sistema (Holland 1998). Esto es lo que se conoce como modelado "de abajo hacia arriba" [bottom-up modeling], donde reglas simples pueden dar lugar a patrones de comportamiento colectivo de alto nivel de complejidad (Axelrod 2004).

La simulación basada en agentes resulta pertinente para el abordaje de sistemas complejos caracterizados por alto grado de localismo e interacción, es decir, sistemas no centralizados, no jerárquicos; sino distribuidos. Los agentes, que son desarrollados e implementado por medio de software, tienen, en general, las siguientes propiedades: son autónomos, interactivos, con conocimiento local, con percepción subjetiva, racionalidad limitada, reactivos y proactivos (Arroyo Méndez y Hassan Collado 2007). El empleo de modelos basados en agentes para la simulación de procesos sociales ha dado lugar al concepto de sociedad artificial (Reynoso 2006).

El punto problemático para las ciencias sociales consiste en que muchos de los modelos de simulación han sido desarrollados con independencia a la teoría política y social, este hecho permite llamar la atención sobre la necesidad de indagar en la legitimidad y pertinencia de las técnicas de simulación en ciencias sociales. El desafío problemático consiste, entonces, en concebir una estrategia metodológica que incluya técnicas de simulación social pero que, al mismo tiempo, contemple la necesidad de construir modelos y simulaciones -y fundamentalmente plataformas informáticas adecuadas para modelizar y simular-, sobre la base de teorías sociales y políticas consistentes, legítimas y relevantes. Sería un error creer que vamos a encontrar en la modelización mulit-agente la solución a todos los problemas metodológicos de las ciencias sociales; pero también sería un equívoco considerar, por prejuicio o ignorancia, que estas metodologías y técnicas no pueden ayudarnos a mejorar la construcción de teoría y el conocimiento de la complejidad organizada propia de nuestras ciencias. Este problemático desafío, al que podemos llamar como simulación social teóricamente informada, es abordado por el proyecto SocLab.

\section{Modelización y simulación de organizaciones sociales con SocLab}

La Sociología de la Acción Organizada (SAO), constituye una teoría sociológica ampliamente probada y fundamentada en el marco de la escuela francesa de sociología de las organizaciones (Crozier 1964; Crozier y Friedberg 1977; Friedberg 1993). Para la SAO, las organizaciones son configuraciones sociales generadas por las relaciones que los actores organizacionales establecen entre sí. Estos actores están dotados de una racionalidad limitada y movilizan sus recursos para disponer de su poder con el fin de preservar o incrementar su autonomía y su capacidad de acción en la organización. El poder de un actor resulta de su capacidad de dominio y control sobre una o más zonas de incertidumbre; es decir, de un recurso necesario para la acción de otros actores. Este dominio le permite al actor fijar, en cierta 
medida, los términos de intercambio en la relación con otros actores (Roggero y Sibertin-Blanc 2008).

Un sistema de acción concreto (SAC) puede ser definido como una configuración social relativamente estabilizada, estructurada por relaciones de poder y constituida por el conjunto de los actores, sus alianzas, sus relaciones y sus formas de regulación, en un contexto organizacional determinado. Todo SAC constituye un contexto de interacción delimitado que estructura la cooperación de un conjunto de actores. Numerosos procesos y fenómenos sociales pueden ser conceptualizados en términos de un SAC; por ejemplo, organizaciones, políticas públicas, procesos de cooperación, relaciones (sociales, políticas, económicas, medioambientales) entre distintos actores en espacios geográficos y temporales determinados (Roggero, et al. 2007).

El equipo interdisciplinario de investigación conformado por sociólogos del LEREPS e informáticos del IRIT de la Universidad de Toulouse 1, ha procedido a formalizar matemáticamente la Sociología de la Acción Organizada. La formalización de la SAO, en conjunción con los modelos de Sistemas Multi-Agentes (SMS), permitió elaborar un meta-modelo de un Sistema de Acción Concreto (SAC) que resulte aplicable para modelizar organizaciones sociales del mundo real y para simular su funcionamiento. Este trabajo dio lugar al desarrollo de una plataforma informática dedicada al estudio de organizaciones sociales denominada SocLab (Sociology Laboratory) (Maillard 2008).

La plataforma SocLab permite modelar organizaciones sociales, analizar su estructura y simular el comportamiento de sus actores, con la finalidad de construir escenarios futuros alternativos de un sistema de acción (SAC) y evaluar su aceptabilidad social (Sibertin-Blanc, et al. 2010).

En primer lugar, la modelización de organizaciones sociales concretas mediante SocLab permite efectuar un análisis de la estructura de una organización social. Este análisis consiste en dar cuenta del conjunto de elementos y relaciones que definen la configuración o el estado actual de una organización social concreta. En otros términos, el estudio analítico de las propiedades estructurales de un sistema social concreto permite dar cuenta de los actores, sus alianzas, sus relaciones, sus recursos, sus capacidades de acción, la autonomía de cada uno de ellos, las relaciones de poder, así como también el nivel de satisfacción global del sistema.

En segundo término, la simulación de una organización social permite dar cuenta de los cambios de estado de un sistema de acción; es decir, los procesos dinámicos de un sistema a lo largo del tiempo y los puntos en los que éste se regula y estabiliza. De este modo, la simulación del comportamiento de actores permite introducir cambios potenciales en el modelo de una organización y generar escenarios futuros alternativos. Estos escenarios constituyen los estados posibles que el sistema podría asumir. La exploración analítica de los estados posibles permite calcular el nivel de satisfacción y poder de cada actor, en función de los cambios introducidos por medio de la simulación.

Es por estas razones que la modelización y simulación de organizaciones sociales mediante SocLab constituye una herramienta metodológica de importancia para 
estimar la aceptabilidad social de políticas públicas orientadas a la transformación de sistemas sociales concretos. El conocimiento producido por SocLab contribuye a la deliberación y toma de decisiones por parte de los actores de gobernanza en los procesos de formulación y aplicación de políticas.

La plataforma SocLab ha sido aplicada en diversos proyectos de investigación interdisciplinarios sobre problemática ambiental. Entre estas aplicaciones se destaca el proyecto europeo Life "Concert'eau" (2006-2009), cuyo objetivo fue la medición de la aceptabilidad socio-organizacional de políticas agro-ambientales destinadas a reducir la polución de las aguas provocada por las explotaciones agrícolas en el departamento de Gers; un territorio ubicado en el sud-oeste de Francia que presentaba una vulnerabilidad ambiental específica derivada de la presencia de nitrato (Adreit, et al. 2009).

Uno de los objetivos centrales del proyecto era mostrar la factibilidad de involucrar a los actores agrícolas del territorio para que propongan nuevas prácticas destinadas a la preservación del agua y del medio ambiente, y se comprometan en su implementación. De este modo, se incrementaba la efectividad de aplicación de las nuevas medidas.

Primero, se realizó una investigación empírica de campo para relevar los datos que serían utilizados para modelizar el sistema social con SocLab. Cabe destacar que el sistema agro-ambiental en cuestión no se presentaba como una organización formal, sino como un sistema de acción difuso en donde había una pluralidad heterogénea de actores involucrados. En una segunda fase, se procedió a simular distintos escenarios futuros en función de las medidas propuestas por los actores. Se realizó un análisis del nivel de satisfacción y poder de cada actor en cada uno de los escenarios simulados. Finalmente, se procedió a la evaluación de la aceptabilidad social de las medidas propuestas considerando cada actor en particular y al sistema social en su conjunto. Este proyecto de investigación constituyó una validación empírica importante del marco teórico y metodológico de la plataforma SocLab para la evaluación cuantitativa de la factibilidad social de políticas agrícolas orientadas a la gobernanza de los recursos hídricos y el medioambiente.

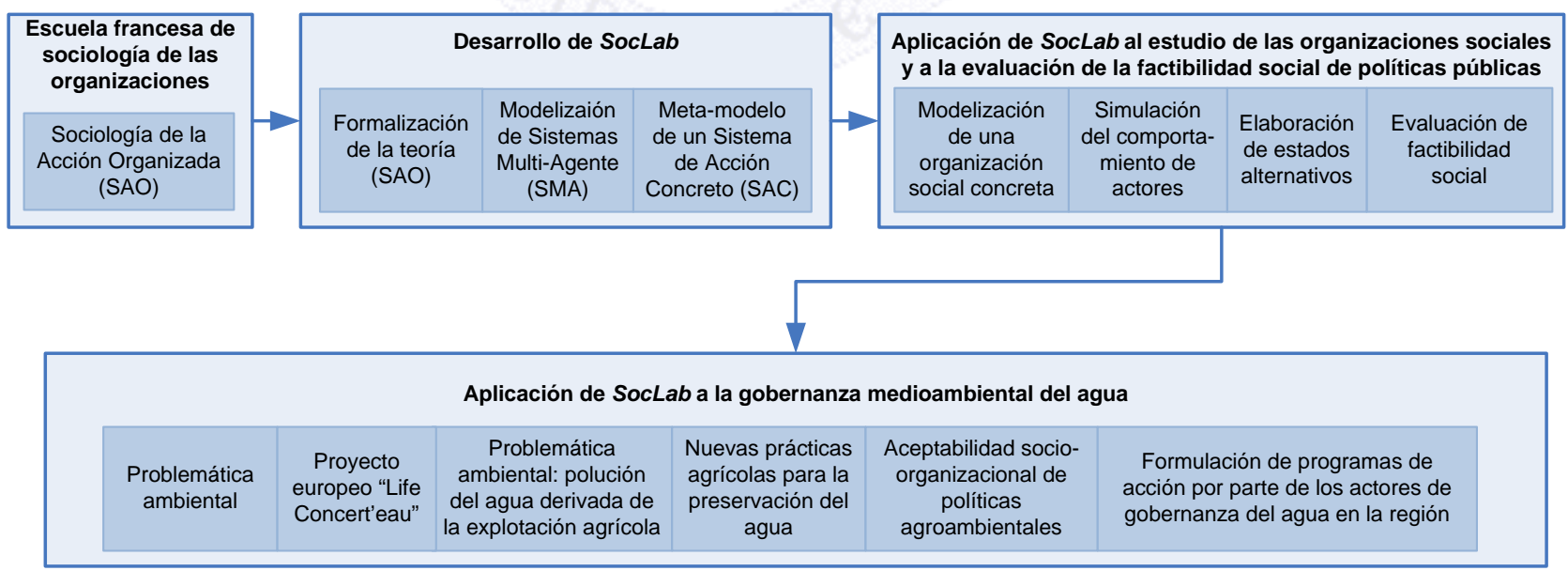




\section{Conclusiones}

El campo de estudios contemporáneos de complejidad se encuentra dividido en dos concepciones distintas y, posiblemente, antagónicas: el pensamiento complejo y las ciencias de la complejidad. Cada una de ellas reclama la pertinencia y legitimidad de un determinado modo de entender la complejidad.

Por un lado, las ciencias de la complejidad se erigen, en la ciencia contemporánea, como una mirada estrictamente científica sobre un nuevo tipo de problemas y objetos de estudio: los sistemas complejos. No obstante, este modo de entender la complejidad parece seguir engrilletado en los problemas epistemológicos heredados de concepciones cientificistas, como puede ser el positivismo lógico en el siglo XX. Esto obedece a la preocupación por establecer un criterio de demarcación rígido entre el estudio científico de la complejidad y las aproximaciones filosófico-reflexivas. En efecto, he argumentado que el marco epistemológico que sustenta a las ciencias de la complejidad no permite abordar y tematizar problemas fundamentales concernientes al lugar de la ética, la política, los valores y la subjetividad en el quehacer científico. En suma, a pesar de las innovaciones técnico-metodológicas de las ciencias de la complejidad, su forma de producir conocimiento continúa siendo funcional a un concepto hegemónico-dominante de ciencia.

Por otro lado, el pensamiento complejo se afirma como una concepción epistémica alternativa al tradicional modo de entender la ciencia y el conocimiento. Su estrategia metodológica se distancia del concepto estándar de método científico, para reclamar la necesidad y pertinencia de un método que incluya la reflexión crítica y auto-crítica; es decir, postula la inclusión del sujeto cognoscente en su conocimiento. El pensamiento complejo esgrime, así, una teoría de la racionalidad post-clásica, en cuyo marco plantea la necesidad de concebir la unidad complejidad (complementaria y antagonista) de dicotomías reificadas por el pensamiento occidental moderno: razón-afectividad, ciencia-filosofía, hecho-valor, objetivosubjetivo, cuerpo-mente, naturaleza-cultura. De este modo, la propuesta del pensamiento complejo propone una reconfiguración epistemológica tendiente hacia un conocimiento transdisciplinar, en el cual, necesariamente, la ciencia tiene que ser articulada con otras formas de conocimiento. Esto no implica renunciar ni abjurar de la ciencia y del conocimiento científico sino, por el contrario, la necesidad de problematizarlo, criticarlo e incluirlo en un marco de comprensión más rico. En esta línea, el pensamiento complejo desarrolla también una propuesta ética y política que aborda la crítica al modo civilizatorio hegemónico en occidente, como objeto central de su problemática.

Juzgo fundamental valorar críticamente los aportes del pensamiento complejo y de las ciencias de la complejidad en el nivel propio en el que cada una de ellas realiza sus contribuciones. Las ciencias de la complejidad nos brindan herramientas magníficas para abordar de modo concreto el estudio de fenómenos complejos como la auto-organización, la emergencia, la no-linealidad, etc. En este trabajo se ha descripto y analizado la metodología de modelización y simulación computacional de sistemas complejos y una de las técnicas de las ciencias de la complejidad: los modelos basados en agentes. Así mismo, se ha mostrado el valor metodológico de este modo de abordaje en una investigación concreta en base a la plataforma SocLab. 
Es preciso reparar en el punto crucial de este debate. Considero política y humanamente riesgoso, y epistemológicamente inaceptable, creer que sólo con buenas herramientas tendremos lo suficiente para crear una mejor ciencia. Entiendo por mejor ciencia aquélla capaz de abordar los problemas humanos fundamentales y, también, aquélla capaz de ser juzgada en base a criterios ético-políticos vinculados a las necesidades sociales de los pueblos.

Podrá objetarse esta toma de partido epistemológicamente justificada ${ }^{11}$. No obstante, es necesaria una toma de conciencia radical. Una concepción de ciencia tal, que sólo concibe la dimensión epistémica y racional de su práctica pero que es incapaz de abordar los aspectos éticos y políticos de su acción; que tampoco puede problematizar la relación de su quehacer científico con las estructuras políticoeconómicas de la sociedad en la cual se desarrolla; y que juzga sus resultados en base al éxito empírico-manipulativo de su conocimiento; puede ser, en efecto, una ciencia de punta en términos instrumentales pero es una ciencia políticamente inconsciente y peligrosa. En la historia hemos visto ya las consecuencias de esta ciencia-martillo (Marí 1991) consagrada a proveer instrumentos de conocimiento supuestamente neutrales y dejando la responsabilidad ética, social y humana de su aplicación a las instancias políticas, los tecnócratas y los gobiernos. Este concepto de ciencia ha fracasado, puesto que ha engendrado nefastas consecuencias humanas (Auschwitz e Hiroshima) pero también a la degradación de la biósfera terrestre y otras formas de dominio y explotación de los seres humanos y la naturaleza. Una nueva civilización requiere, efectivamente, de una nueva ciencia.

Es ésta la razón cardinal por la cual el dominio científico de la complejidad será siempre parcial y limitado. Es por ello que la apuesta más fundamental para las ciencias contemporáneas consistiría en incluir la potencialidad metodológica de las ciencias de la complejidad en un marco epistémico ampliado a la ética y la política como propone el pensamiento complejo.

\section{Bibliografía}

ADREIT, FRANÇOISE, ROGGERO, PASCAL, SIBERTIN-BLANC, CHRISTOPHE y VAUTIER, CLAUDE 2009. Using Soclab for a Rigorous Assessment of the Social Feasibility of Agricultural Policies. Toulouse, France: Life Project led by Ecobag-ADERA (Association pour le Développement de l'enseignement et de la Recherche auprès des universités, des centres de recherche et des entreprises d'Aquitaine) and AEAG (Water Agency of Adour Garonne), assisted by: Cémagref, the Centro de Recursos Ambiantales de Navarra, CNRS, Enfa, Gramip, Infoterra, INPT, INRA, IRH, Silogic and the University of Toulouse 1.

ARMATTE, MICHEL, (2006), "La Nocion de Modelo en las Ciencias Sociales". Empiria. Revista de Metodologia de Ciencias Sociales 11:33-70.

ARROYO MÉNDEZ, MILLÁN y HASSAN COLLADO, SAMER, (2007), "Simulación de procesos sociales basada en agentes software". Empiria. Revista de Metodologia de Ciencias Sociales 14:139-161.

\footnotetext{
${ }^{11}$ He investigado con profundidad el problema de la dimensión política de la ciencia y de las prácticas científicas en otro trabajo. Al respecto véase Rodríguez Zoya (2010a).
} 
ASHBY, W. R., (1962), Principles of self-organization, En Hinez von Foerster and GW Zopf Jr., Principles of Self-Organization: Transactions of the University of Illinois Symposium, Pergamon Press, New York.

AXELROD, ROBERT, (1997), The complexity of cooperation. Princeton Univeristy Press, Princeton.

AXELROD, ROBERT, (2004), La complejidad de la cooperación. Modelos de cooperación y la colaboración basada en los agentes. $1^{\circ}$ ed. FCE, Buenos Aires.

BERTALANFFY, LUDWING VON (1968), Teoría general de sistemas. Fundamentos, desarrollos, aplicaciones. 2007, $1^{\circ}$ ed. Fondo de Cultura Económica, Buenos Aires, Argentina.

CAPRA, FRITJOF, (1984), El tao de la física. Una exploración de los paralelismos entre la física moderna y el misticismo oriental. 1992, $3^{\circ}$ ed. Editorial Humanitas, Barcelona, España.

CROZIER, MICHEL, (1964), Le phénomène bureaucratique. Seuil, Paris.

CROZIER, MICHEL y FRIEDBERG, ERHARD, (1977), L'Acteur et le système. Dynamiques de l'action collective. Seuil, Paris.

DELGADO DÍAZ, CARLOS, (2004), "The political significance of small things". Emergence: Complexity and Organization 6 (Special Double Issue. Nos. 12):49-54.

FOERSTER, HEINZ VON, (1996), Las semillas de la cibernética. 1996, $2^{\circ}$ ed, Colección terapia familiar. Gedisa, Barcelona.

FRIEDBERG, ERHARD, (1993), Le Pouvoir et la Règle. Dynamiques de l'action organisée. Seuil, Paris.

GARCÍA, ROLANDO, (2000), Capítulo 4: Los procesos cognoscitivos, En Rolando García, El conocimiento en construcción. De las formulaciones de Jean Piaget a la teoría de los sistemas complejos, Gedisa, Barcelona, Pag.95-113.

GARCÍA, ROLANDO, (2006), Sistemas complejos. Conceptos, método y fundamentación epistemológica de la investigación interdisciplinaria. $1^{\circ}$ ed. Gedisa, Barcelona.

GELL-MANN, MURRAY, (1994), El Quark y el Jaguar. Aventuras en lo simple y lo complejo. $4^{\circ} \mathrm{ed}$. Tusquets, Barcelona, España.

GILBERT, NIGEL y TROITZSCH, KLAUS G., (1999), Simulation for the social scientist. $20051^{\circ}$ ed. Open University Press, Milton Keynes.

HOLLAND, JOHN, (1995), Hidden order: How adaptation builds complexity. Perseus Books, Cambridge.

HOLLAND, JOHN, (1998), Emergence. From chaos to order. Reading, MA: AddisonWesley.

IZQUIERDO, LUIS, GALÁN ORDAX, JOSÉ MANUEL, SANTOS, JOSÉ I y OLMO MARTÍNEZ, RICARDO DEL (2008), "Modelado de sistemas complejos mediante simulación basada en agentes y mediante dinámica de sistemas". EMPIRIA. Revista de Metodologia de Ciencias Sociales 16:85-112.

JOHNSON, STEVEN, (2001), Sistemas emergentes. O qué tienen en común hormigas, neuronas, ciudades y software. 2003, $1^{\circ}$ ed, Colección Noema. Fondo de Cultura Económica, Madrid.

MAILLARD, MATTHIAS. 2008. MultiAgents formalisation for the Sociology of the Organized Action. PhD thesis, University of Toulouse, Toulouse, France.

MALDONADO, CARLOS, (2008), "Complejidad y Ciencias Sociales desde el aporte de las Matemáticas Cualitativas". Cinta de Moebio 33:153-170. 
MALDONADO, CARLOS, (2009), "Complejidad de los sistemas sociales: un reto para las ciencias sociales". Cinta de Moebio 36:146-157.

MALDONADO, CARLOS EDUARDO, (2007), Complejidad: ciencia, pensamiento y aplicación. Universidad Externado de Colombia, Buenos Aires, Argentina.

MALDONADO, CARLOS y GÓMEZ CRUZ, NELSON ALFONSO. 2010. Modelamiento y simulación de sistemas complejos. Bogotá: Universidad del Rosario.

MANDELBROT, BENOÎT, (1987), Los objetos fractales. 2006, $6^{\circ}$ ed. Tusquets, Barcelona, España.

MARÍ, ENRIQUE E., (1991), "Ciencia y ética. El modelo de la ciencia martillo". Doxa, Cuadernos de Filosofía del Derecho 10:319-327.

MATURANA, HUMBERTO y VARELA, FRANCISCO, (1972), Autopoietic system. Facultad de Ciencias, Santiago de Chile.

MORIN, EDGAR, (1977), El Método I. La naturaleza de la naturaleza. 2001, $1^{\circ} \mathrm{ed}$. Cátedra, Madrid.

MORIN, EDGAR, (1980), El Método II. La vida de la vida. 2002, $5^{\circ}$ ed. Cátedra, Madrid.

MORIN, EDGAR, (1986), El Método III. El conocimiento del conocimiento. 2002, $4^{\circ}$ ed. Cátedra, Madrid.

MORIN, EDGAR, (1990), Introducción al Pensamiento Complejo. 2001, $4^{\mathrm{a}}$ reimpresión, $1^{\circ}$ ed. Gedisa, Barcelona.

MORIN, EDGAR, (1991), El Método IV. Las ideas. 1998, $2^{\circ}$ ed. Cátedra, Madrid.

MORIN, EDGAR, (2001), El Método V. La humanidad de la humanidad. La identidad humana. 2003, $1^{\circ}$ ed. Cátedra, Madrid.

MORIN, EDGAR. 2008. Complejidad restringida, complejidad general Biblioteca Virtual Participativa de la Complejidad, 2004a [cited 19-11 2008]. Available from

http://www.pensamientocomplejo.com.ar/documento.asp?Estado=VerFicha\&l dDocumento $=237$.

MORIN, EDGAR. 2008. Epistemología de la complejidad Biblioteca Virtual Participativa de la Complejidad, 2004b [cited 19-11 2008]. Available from http://www.pensamientocomplejo.com.ar/documento.asp?Estado=VerFicha\&l dDocumento=71.

MORIN, EDGAR, (2004c), La Méthode VI. Éthique. 2004, $1^{\circ}$ ed. Seuil, Paris.

MORIN, EDGAR y BRIGITTE KERN, ANNE, (1993), Tierra-Patria. 1999, $2^{\circ}$ ed. Nueva Visión, Buenos Aires.

NEUMANN, JOHN VON, (1966), Theory of Self-Reproducing Automata. Univeristy of Illinois Press, Urbana.

NEUMANN, JOHN VON, (1968), The General and Logical Theory of Automata. Aldine, Chicago.

PIAGET, JEAN, (1978), La equilibración de las estructuras cognitivas. Problema central del desarrollo. $1^{\circ}$ ed. Siglo XXI, México.

PRIGOGINE, ILYA y NICOLIS, GRÉGOIRE, (1987), La estructura de lo complejo. 1997, $1^{\circ}$ ed. Alianza, Madrid.

REYNOSO, CARLOS, (2006), Complejidad y caos. Una exploración antropológica. $1^{\circ}$ ed. Editorial SB, Buenos Aires, Argentina.

REYNOSO, CARLOS, (2009), Modelos o metáforas. Crítica del paradigma de la complejidad de Edgar Morin. $1^{\circ}$ ed. Editorial SB, Buenos Aires.

RODRÍGUEZ ZOYA, LEONARDO, (2010a), Complejidad de la relación entre ciencia y valores. La significación política del conocimiento científico. $1^{\circ}$ ed. Vol. 19. 
Instituto de Investigaciones Gino Germani, Universidad de Buenos Aires, Buenos Aires.

RODRÍGUEZ ZOYA, LEONARDO, (2010b), "Hacia una epistemología política: la tensión entre ciencia y política en la filosofía de la ciencia del positivismo lógico". A Parte Rei. Revista de Filosofía 69 (Mayo):1-17.

ROGGERO, PASCAL, SIBERTIN-BLANC, MAILLARD, MATTHIAS, ADREIT, FRANÇOISE, VAUTIER, CLAUDE y SANDRI, SANDRA. 2007. Formalisation, modélisation et simulation orientée agents des systèmes d'action concrets : approche complexe de la sociologie de l'action organisée. Tolouse, France: Rapport de recherche, Université des Sciences Sociales.

ROGGERO, PASCAL y SIBERTIN-BLANC, CHRISTOPHE, (2008), "Quand des sociologues rencontrent des informaticiens : essai de formalisation des systèmes d'action concrets". Nouvelles Perspectives en Sciences Sociales 3 (2):41-81.

SIBERTIN-BLANC, CHRISTOPHE, ADREIT, FRANÇOISE, CHAPRON, PAUL, EL GEMAYEL, JOSEPH, MAILLIARD, MATTHIAS, ROGGERO, PASCAL y VAUTIER, CLAUDE, (2010), "Compte-rendu d'une recherche interdisciplinaire entre sociologues et informaticiens: de la sociologie de l'action organisée au logiciel SocLab". Hermès Science Publications 29 (3).

SOTOLONGO, PEDRO y DELGADO DÍAZ, CARLOS JESUS, (2006), La revolución contemporánea del saber y la complejidad social. Hacia unas ciencias sociales de un nuevo tipo. $1^{\circ} \mathrm{ed}$, Campus Virtual. CLACSO Libros, Buenos Aires.

THOM, RENE, (1976), "Structural Stability, Catastrophe Theory, and Applied Mathematics: The John von Neumann Lecture, 1976". SIAM Review 19 (2):189-201.

VILAR, SERGIO, (1997), La nueva racionalidad. Comprender la complejidad con métodos transdisciplinarios. $1^{\circ}$ ed, Colección Nueva Ciencia. Kairós, Barcelona, España.

WEAVER, WARREN, (1948), "Science and complexity". American Scientist (36):536.

WIENER, NORBERT, (1985), Cibernetica. O el control y la comunicación en animales y máquinas. $1^{\circ} \mathrm{ed}$. Tusquets, Barcelona, España. 